\title{
THEMATISCHER SCHWERPUNKT 2014: Emotionen, Routinen
}

Zwischen Emotion und Routine besteht ein in vielerlei Hinsicht interessantes Spannungsfeld, das unter literatur-, kultur- oder sprachwissenschaftlichem Blickwinkel untersucht werden kann. Der Emotionsbegriff evoziert eine gewisse Dynamik; in ihm laufen verschiedene Formen menschlichen Wahrnehmens und der Reaktion darauf zusammen. Das Ringen um einen angemessenen sprachlichen Ausdruck einzelner Facetten der menschlichen Empfindungen, Emotionen und Gefühle ist schon immer eine Herausforderung für Schriftsteller und Dichter, aber auch für Psychologen und Lexikologen gewesen, denn, wie einst die Linguistin Anna Wierzbicka trefflich sagte, „Gefühle sind etwas, was man fühlt und nicht in Worten erlebt"“, was auch mutatis mutandis auf die Emotionen ausgedehnt werden kann. Dagegen steht allerdings Friedrich Nietzsches Aussage: „Hinter den Gefühlen stehen Urteile und Wertschätzungen, welche in der Form von Gefühlen (Neigungen, Abneigungen) uns vererbt sind.“" Und Ute Frevert schreibt: „Gefühle drängen geradezu darauf, sich in Form und Symbol auszudrücken, und schaffen auf diese Weise einen Verständigungsraum, der die oder den Einzelnen mit anderen vergemeinschaftet. Sie sind vorsprachlich oder körperkonzentriert, können aber in Worte gefasst und diskursiv reguliert werden. Sie sind Teil eines individuellen, gleichsam privaten Zugangs zur Welt. Doch sie bilden zugleich Trajektoren kollektiver, öffentlicher Welterfahrung und Weltveränderung. Eine kulturwissenschaftlich angeleitete Geschichte der Gefühle führt demnach in einen zentralen Problembereich historiographischer Analysen, nämlich in das Verhältnis von Individualität und Soziabilität, zwischen Person und Umwelt, Privatheit und Öffentlichkeit.“2 Günter Oesterle brachte die „Sprachwerdung des Gefühls“ aus kultur- und literaturgeschichtlicher Perspektive in die Debatte. ${ }^{3}$ Er denkt dabei vor allem an die Literatur des 18. Jhd.s (Herder sagte bekanntlich, Descartes umdrehend: „Ich fühle mich! Ich bin!“) ${ }^{4}$, doch

1 FrIEDRICH NiETZSCHE (1971): Werke. Kritische Gesamtausgabe. Hrsg. von Giorgio Colli und Mazzino. Bd. V, 1: Morgenröthe. Berlin/New York, 39f.

2 Zit. nach Hugh Ridley / AnNe Fuchs / SAbine StrüMPER-Krobb (eds.) (2003): Sentimente, Gefühle, Empfindungen, Zur Geschichte und Literatur des Affektiven von 1770 bis heute. Würzburg, 20f.

3 Zit. nach RIDLEY / FUCHS / STRÜMPER-KROBB, $45 \mathrm{f}$.

4 Johann GotTFRIED Herder (1994): Schriften zu Philosophie, Literatur, Kunst und Altertum 1774-1787. Hrsg. von Jürgen Brummack und Martin Bollacher. 
seit dieser Zeit ist eine gesteigerte Aufmerksamkeit für Emotionen, Affekte, Empfindungen, Gefühle, Stimmungen, Gemütszustände zu beobachten, wobei auch zunehmend - insbesondere in neuerer Zeit - auf sogenannte negative emotionale Reaktionen wie Furcht, Hass und Ekel fokussiert wird. Neue Gesichtspunkte haben nicht nur die Psychoanalyse, sondern auch die Genderforschung ins Spiel gebracht ${ }^{5}$, u. a. das Problem der Leiblichkeit des Gefühls. ${ }^{6}$ Thomas Anz gebraucht sogar den Begriff ,emotional turn“.7

Während in der Fachsprache der Mediziner und Psychologen Emotionen und Gefühle wohlunterschiedene Begriffe zu sein pflegen, überlagern sich in der Alltagssprache und auch in der Sprache der Belletristik beide Sphären zumeist. Man gebraucht hier die verschiedensten Bezeichnungen. Deren Bezüge und gegenseitigen Beeinflussungen wären zu hinterfragen, aber auch das Verhältnis zwischen Fach- und Alltagssprache, präziser: zwischen der Art und Weise, wie diese reflektiert und artikuliert werden. ${ }^{8}$

Auf dem anderen Pol stehen Routinen. Meist (zu Unrecht) als Antithese der Kreativität angesehen, sind sie in Wirklichkeit ein wichtiger stabilisierender Faktor vieler Lebensbereiche. Durch ihre Systematizität, Wiederholbarkeit und Prädiktabilität setzen sie gewisse Erwartungsrahmen, die den Lauf der Dinge in der Welt überschaubar(er) und/oder handhabungsfähig(er) machen. Literaturwissenschaftler haben diesen Gesichtspunkt z. B. in ihren Erörterungen über die Rolle des Alltags in der Literatur des 19. und 20. Jhd.s problematisiert. In der Überschrift, die für das Schwerpunktthema gewählt wurde,

Frankfurt (M.), 236 (es handelt sich um den Essay Zum Sinn des Gefühls). Siehe auch das Buch des portugiesisch-amerikanischen Neurologen ANTONIO R. DAMASIO (2000): „Ich fühle, also bin ich“. München.

5 Ein Beispiel hierfür wäre: MANuEl BorutTa / NinA VerheYen (eds.) (2010): Die Präsenz der Gefühle - Männlichkeit und Emotion in der Moderne. Bielefeld.

6 ThOMAs ANZ erklärt in seiner Rezension Die Wissenschaften und die Gefühle. Skizzen und Literaturhinweise u.a.: „Auch die empfindsame Verschriftlichung von Gefühlen mag auf die zentrale Bedeutung des Körpers als Zeichenträger emotionaler Aussagen nicht verzichten." Siehe http://www.literaturkritik.de/ public/rezension.php?rez_id=3277 (12.7.2012).

7 Siehe hierzu: ThOMAs ANz: Emotional Turn? Beobachtungen zur Gefühlsforschung: http://www.literaturkritik.de/public/rezension.php?rez_id=10267 (12.7.2012).

$8 \quad$ Hierzu als Einstieg: LuDWIG JäGER (ed.) (1988): Zur Historischen Semantik des deutschen Gefühlswortschatzes. Aspekte, Probleme und Beispiele einer lexikographischen Erfassung. Aachen. 
steht ein nicht disjunktives Komma. Somit können Emotion und Routine in Bezug aufeinander betrachtet werden, es sei denn, dass der Autor sein Augenmerk nur auf eine der beiden Sphären richten möchte.

Die Convivium-Redaktion lädt zur Auseinandersetzung mit dem vorgeschlagenen Rahmenthema ein. Der erhoffte Ertrag ist eine Reihe von Aufsätzen in den Sparten Literatur-, Kultur- und Sprachwissenschaft, ferner Psycho- und Soziolinguistik, in denen die Facetten der uns interessierenden Erscheinungen von verschiedenen Seiten beleuchtet werden. Autoren, die sich zu diesem thematischen Schwerpunkt äußern möchten, werden gebeten, der Redaktion den vorläufigen Titel des geplanten Textes bis spätestens zum 30. Juni 2013 mitzuteilen. Die endgültige Fassung erbeten wir uns bis zum 30. November 2013 (CD + ein Ausdruck). Die Richtlinien zur Einrichtung des druckfertigen Manuskripts sind der Website www.convivium.pl, und die Redaktionsanschrift ist dem Impressum zu entnehmen.

$\mathrm{LC} / \mathrm{KS}$ 